\title{
Mortality in patients with epilepsy: 40 years of follow up in a Dutch cohort study
}

\author{
D P Shackleton, R G J Westendorp, D G A Kasteleijn-Nolst Trenité, J P Vandenbroucke
}

Department of Clinical Epidemiology, Faculty of Medicine, Leiden University, The

Netherlands D P Shackleton R G J Westendorp J P Vandenbroucke

Instituut voor Epilepsiebestrijding, Heemstede, The

Netherlands

D P Shackleton

D G A Kasteleijn-Nolst

Trenité

Correspondence to: Dr D G A Kasteleijn-Nolst Trenité, Instituut voor Epilepsiebestrijding, Meer en Bosch, De Cruquiushoeve, Achterweg 5, $2103 \mathrm{SW}$

Heemstede, The

Netherlands.

Received 23 December 1997 and in final form 30 October 1998

Accepted 20 November 1998

\begin{abstract}
To investigate the extent of and the causes of excess mortality in patients with epilepsy, mortality was studied in a cohort of patients with newly diagnosed epilepsy over an extended follow up period. All patients (both inpatients and outpatients) of the Instituut voor Epilepsiebestrijding in Heemstede, the Netherlands between 1953 and 1967 were included in the study. Complete follow up was obtained for 1355 patients, 746 men and 609 women. The mean follow up was 28 years (range 6 months-41 years). In total, 38665 person years were surveyed, in which 404 patients died. All cause mortality was threefold increased (risk ratio (RR) 3.2; 95\%CI 2.93.5), and was only slightly higher for men than for women. Mortality was highest under 20 years of age (RR 7.6; 95\%CI 6.58.7 ), and during the first 2 years of follow up (RR 16; 95\%CI 12-20).

Mortality directly related to epilepsy accounted for 18 of the 53 deaths in the first 2 years after diagnosis, which is equivalent to an incidence rate of 6.8 per 1000 person-years (95\% CI 4.1-10). After 2 years 110 of the 351 deaths could be attributed to epilepsy itself, or were epilepsy related, with an incidence rate of 3.1 per 1000 person-years (95\% CI 2.5-3.6). The data presented suggest that the increased mortality risk in patients with epilepsy is attributable in part to epilepsy itself, and is predominantly present at younger age and early after diagnosis. However, the absolute risk is moderate.

(F Neurol Neurosurg Psychiatry 1999;66:636-640)
\end{abstract}

Keywords: epilepsy; epidemiology; mortality; standard mortality ratio

Contrary to Gower's dictum, ${ }^{1}$ it is now generally accepted that mortality of patients with epilepsy is higher than that of the general population. ${ }^{2-4}$ In 1910 Munson reported that "...the disease carries with it very grave dangers, the span of life is shortened, and there is a special liability to certain diseases and to traumas". ${ }^{2}$ This excess mortality ranges from twofold to 10-fold, as shown by various studies carried out this century. ${ }^{5-15}$ None the less, the extent of and causes of this increase remain unclear. Type of epilepsy, patient selection, duration of follow up, and chronology of study implementation are all determinants that possibly contribute to the differences between the estimates.

Data from contemporary studies, comprising newly diagnosed patients with epilepsy, show excess mortality in the first decade of life and the first few years after diagnosis. Excess mortality is predominantly present in patients with symptomatic epilepsy, whereas excess mortality is increased only slightly in patients with idiopathic or cryptogenic epilepsy. ${ }^{10} 1314$ However, cohorts from institutions and specialist centres have consistently shown a significant excess mortality from the epilepsy itself..$^{912}$ None the less, these findings have led authors to conclude that excess mortality can largely be explained by the predisposing causes of the seizures, rather than the seizures themselves. ${ }^{13}$ This conclusion, however, demands a sound classification method for epilepsy seizures and syndromes, and the present classification criteria proposed by the International League Against Epilepsy ${ }^{16}$ are not without problems. ${ }^{17}$

In this paper we investigated the hypothesis that excess mortality is in part due to epilepsy itself. Rather than determining mortality dependent on the types of epilepsy and epilepsy aetiology, we investigated causes of death directly, comparing mortality which could be ascribed to underlying causes, and mortality due to epilepsy itself or related to epilepsy. We were given the special opportunity of forming a large cohort of newly diagnosed epileptic patients with an extensive follow up of up to 40 years.

\section{Methods}

PATIENT SERIES

To obtain the data for those patients who had visited the Instituut voor Epilepsiebestrijding for the first time between 1953 and 1967, all 22000 patient records (all inpatient and outpatient records were preserved in the archives of the Institute) were reviewed. These records were screened to identify patients with newly diagnosed epilepsy. Identification was based on the diagnosis made by a neurologist at the institute, within 1 year of the first consultation. The diagnosis was primarily based on a detailed patient history, and routine EEG 
recordings and the classification methods of Penfield and Jasper ${ }^{18}$ were employed. If the initial diagnosis seemed uncertain-for example-psychogenic seizures, the original clinical history and EEGs were reviewed by one of us (DKNT) and reclassified either as certain epilepsy or as no or uncertain epilepsy. Using patient histories, all possible care was taken to ensure that patients with remote symptomatic unprovoked seizures were excluded from the cohort.

Records were identified between September 1993 and August 1994. The demographic data extracted from the patient records included: sex, date of birth, date of first admission, and addresses of both the time of first and last consultation. All patients were followed up until December 1994.

ANALYSIS OF MORTALITY

All cause mortality and mortality from specific causes were compared with that of the general Dutch population. The primary and secondary causes of death were assessed by linking the death certificate numbers obtained from the civic registries to the causes of death recorded by the physician of the Dutch Central Bureau of Statistics. The expected number of deaths among patients with epilepsy was calculated by multiplying the number of person-years per category of age, sex, and calendar period with the corresponding mortality rates of the Dutch population. The standardised mortality ratio (SMR) was calculated by dividing the observed number of deaths by the expected number of deaths. The $95 \%$ confidence interval (95\% CI) of the SMR was calculated, assuming a Poisson distribution for the observed number of deaths.

Besides the overall SMR, the mortality ratios in the first 2 years of follow up were compared with those during the subsequent follow up. The assumption was made that most deaths in patients with symptomatic epilepsy of both acute and remote forms occur in the first 2 years after diagnosis. ${ }^{1319} 20$ The effect of age at diagnosis on mortality was studied by comparing patients under 20 years of age at diagnosis with those over 20 years of age, as about $60 \%$ of all epileptic patients present before the age of 20 years. $^{9} 1021$

The SMRs of specific causes of death were determined and tabulated according to follow up duration and age at entry. The specific causes of death used in the analysis were malignancies, respiratory diseases, cardiovascular diseases, all external causes, and the CNS. As brain tumours, cerebrovascular disease, and diseases of the CNS are causes of death that are related to the underlying causes of epilepsy, ${ }^{20}$ SMRs from these causes were analysed with those deaths attributable to epilepsy or epilepsy itself excluded. Accidental death was also considered in the analysis, as it might directly or indirectly be related to epilepsy. ${ }^{20}$ Suicide, a disquieting and potentially preventable cause of death, may also be related to epilepsy, an increased risk being reported in some studies, ${ }^{89}$ but not in others. ${ }^{10}{ }^{13}$ Both status epilepticus and sudden death in epilepsy (SUDEP) could not be distinguished with certainty from other deaths directly related to epilepsy. These causes of death are classified as death from epilepsy (personal communication, Dutch Central Bureau of Statistics).

\section{Results}

CHARACTERISTICS OF THE STUDY POPULATION

Of the more than 20000 patient records that we reviewed, roughly 2000 patients visited the institute for the first time in the period 1953-67. From these, less than 400 were diagnosed as not having epilepsy; 51 of a further 105 patients, for whom the initial diagnosis was uncertain, were reclassified as certain epilepsy. The final cohort consisted of 1455 newly diagnosed epilepsy patients, nearly all of whom were white. Using the civil registries, only 47 men and 53 women were lost in follow up, primarily due to refusal of specific community registries to give appropriate information. The lost to follow up cases were excluded from the analysis. Complete follow up at the end of 1994 was obtained for 1355 patients (93\%). Of these, 746 were men and 609 were women. The mean age on entry of the study was 19 years (range 6 months-70 years). The age distribution was similar for both sexes.

\section{ALL CAUSE MORTALITY}

The mean follow up duration was 28 years (range 6 months-41 years), and 3665 personyears were surveyed. During this period 404 patients died, compared with 128 deaths from all causes that would have been expected, based on mortality rates from the Dutch population. The crude all cause SMR for the study group was calculated as $3.2(95 \%$ CI $2.9-3.5)$. The SMR for men was 3.6 (95\% CI 3.1-4.0), and for women 2.6 (95\% CI: 2.2-3.0).

The SMRs according to years of follow up are shown in table 1. The SMR during the first years of follow up is markedly increased, declining to a twofold increase after 15 years of follow up. To assess the mortality in patients with genuine epilepsy by excluding the mortality that possibly could be attributed to underlying diseases, an analysis was performed excluding data of the first 2 years of follow up. This resulted in an SMR for all patients of 2.8 (95\% CI 2.5-3.1). The SMRs by age at entry are summarised in table 2; the SMR is markedly high in the younger age groups. A second, lower peak is evident in the 60-69 age

Table 1 All cause mortality in patients with epilepsy according to years of follow up

\begin{tabular}{lllll}
\hline Follow up $(y)$ & Person-years & Obs & SMR & $95 \% C I$ \\
\hline $0-1$ & 2646 & 53 & 16 & $11-20$ \\
$2-4$ & 3850 & 37 & 7.0 & $4.7-9.2$ \\
$5-9$ & 6116 & 78 & 6.9 & $5.4-8.4$ \\
$10-14$ & 5795 & 53 & 3.9 & $2.8-5.0$ \\
$15-19$ & 5588 & 40 & 2.4 & $1.6-3.1$ \\
$20-24$ & 5358 & 43 & 2.2 & $1.6-2.9$ \\
$24-29$ & 4834 & 46 & 2.0 & $1.4-2.6$ \\
$30-34$ & 3106 & 40 & 2.0 & $1.4-2.6$ \\
$35-41$ & 1372 & 14 & 0.9 & $0.4-1.4$ \\
$0-41$ & 38665 & 404 & 3.2 & $2.9-3.5$
\end{tabular}

Obs=Observed number of deaths; SMR=observed number of deaths divided by expected number of deaths. 
Table 2 All cause mortality in patients with epilepsy by age group at entry

\begin{tabular}{lllllll}
\hline Age $(y)$ & Obs & $\begin{array}{l}\text { Males SMR } \\
(95 \% \text { CI })\end{array}$ & Obs & $\begin{array}{l}\text { Females SMR } \\
(95 \% \text { CI })\end{array}$ & Obs & $\begin{array}{l}\text { Both SMR } \\
(95 \% \text { CI })\end{array}$ \\
\hline $0-9$ & 66 & $12(9-15)$ & 33 & $13(9-18)$ & 99 & $13(10-15)$ \\
$10-19$ & 51 & $4.9(3.6-6.3)$ & 27 & $5.4(3.5-7.6)$ & 78 & $5.0(4.0-6.2)$ \\
$20-29$ & 51 & $4.5(3.3-5.8)$ & 20 & $2.9(1.8-4.4)$ & 71 & $3.9(3.0-4.9)$ \\
$30-39$ & 43 & $2.5(1.8-3.3)$ & 22 & $2.2(1.4-3.3)$ & 65 & $2.4(1.8-3.0)$ \\
$40-49$ & 29 & $1.5(1.0-2.1)$ & 16 & $1.3(0.7-2.1)$ & 45 & $1.4(1.0-1.9)$ \\
$50-59$ & 19 & $2.8(1.7-4.3)$ & 9 & $0.8(0.4-1.4)$ & 28 & $1.6(1.0-2.2)$ \\
$60-69$ & 6 & $1.8(0.6-3.6)$ & 10 & $2.4(1.1-4.1)$ & 16 & $2.1(1.2-3.3)$ \\
$70+$ & 1 & $1.1-$ & 1 & $0.8-2-2.12$ & 2 & $0.9(0.1-2.6)$ \\
All & 266 & $3.6(3.1-4.0)$ & 138 & $2.6(2.2-3.0)$ & 404 & $3.2(2.9-3.5)$ \\
\hline
\end{tabular}

Obs=Observed number of deaths; SMR=observed number of deaths divided by expected number of deaths.

Table 3 All cause mortality in patients with epilepsy by 5 year age bands

\begin{tabular}{lllllll}
\hline Age $(y)$ & Obs & $\begin{array}{l}\text { Males SMR } \\
(95 \% C I)\end{array}$ & Obs & $\begin{array}{l}\text { Females SMR } \\
(95 \% \text { CI) }\end{array}$ & Obs & $\begin{array}{l}\text { Both } \text { SMR }(95 \% \\
\text { CI) }\end{array}$ \\
\hline $0-4$ & 11 & $39(19-67)$ & 9 & $64(29-114)$ & 20 & $48(29-71)$ \\
$5-9$ & 18 & $43(25-65)$ & 5 & $26(8.0-55)$ & 23 & $38(24-55)$ \\
$10-14$ & 11 & $20(10-34)$ & 9 & $35(16-64)$ & 20 & $25(15-37)$ \\
$15-19$ & 26 & $16(10-23)$ & 7 & $12(4.8-23)$ & 33 & $15(10-21)$ \\
$20-24$ & 15 & $6.5(3.6-10)$ & 7 & $8.8(3.4-17)$ & 22 & $7.1(4.4-11)$ \\
$25-29$ & 19 & $8.6(5.1-13)$ & 10 & $11(5.0-18)$ & 29 & $9.2(6.1-13)$ \\
$30-34$ & 17 & $6.9(3.9-11)$ & 12 & $9.6(4.9-16)$ & 29 & $7.8(5.2-11)$ \\
$35-39$ & 19 & $5.8(3.5-8.8)$ & 12 & $6.2(3.1-10)$ & 31 & $6.0(4.0-8.3)$ \\
$40-44$ & 17 & $3.8(2.2-5.8)$ & 7 & $2.6(1.0-5.0)$ & 24 & $3.4(2.1-4.9)$ \\
$45-49$ & 15 & $2.5(1.4-3.9)$ & 5 & $1.4(0.4-2.9)$ & 20 & $2.1(1.3-3.1)$ \\
$50-54$ & 21 & $2.9(1.7-4.2)$ & 5 & $1.3(0.4-2.7)$ & 26 & $2.3(1.5-3.3)$ \\
$55-59$ & 18 & $2.1(1.2-3.2)$ & 15 & $3.5(1.9-5.5)$ & 33 & $2.5(1.7-3.5)$ \\
$60-64$ & 22 & $2.4(1.5-3.5)$ & 7 & $1.4(0.5-2.6)$ & 29 & $2.0(1.3-2.8)$ \\
$65-69$ & 15 & $1.7(0.9-2.7)$ & 8 & $1.3(0.6-2.4)$ & 23 & $1.6(1.0-2.3)$ \\
$70-74$ & 7 & $0.8(0.3-1.6)$ & 6 & $0.9(0.3-1.9)$ & 13 & $0.9(0.5-1.4)$ \\
$75+$ & 15 & $1.9(1.0-2.9)$ & 15 & $1.0(0.5-1.5)$ & 29 & $1.2(0.8-1.7)$ \\
All & 266 & $3.6(3.1-4.0)$ & 138 & $2.6(2.2-3.0)$ & 404 & $3.2(2.9-3.5)$ \\
\end{tabular}

$\overline{\text { Obs=Observed number of deaths; } S M R=\text { observed number of deaths divided by expected number }}$ of deaths.

group. The SMRs for 5 year age bands of both sexes were also calculated, and are given in table 3 .

To evaluate referral patterns at the time of diagnosis, we compared the SMRs of the 481 patients living within a radius of $30 \mathrm{~km}$ of the Epilepsy Institute with the SMRs of the 874 patients living outside this region at the time of diagnosis. The SMR in patients in the Heemstede region was only slightly lower (SMR 2.8; $95 \%$ CI 2.3-3.3) than the SMR of those patients from the outlying regions (SMR 3.4; 95\% CI 3.0-3.8). The calendar period in which the patient was diagnosed with epilepsy had little effect on the SMRs. The SMR of the 755 patients diagnosed in the period 1953-60 was 3.3 (95\% CI 2.8-3.9), and was similar to the SMR in the 600 patients from the period 1961-1967 (SMR 3.1; 95\% CI 2.7-3.4).

\section{SPECIFIC CAUSES OF DEATH}

The various causes of death, dependent on follow up and age at diagnosis, is presented in table 4 . In the first 2 years of follow up, malignant CNS neoplasms, cerebrovascular diseases, and other CNS diseases, possibly accountable for acute symptomatic epilepsy, were responsible for $19 \%$ of the mortality (10 of 53 deaths). After the first 2 years of follow up, these underlying causes were responsible for $15 \%$ of the mortality (52 of 351 deaths). Malignant non-CNS neoplasms accounted for only one death within the first 2 years, but also for $17 \%$ of the mortality (58 deaths) in the remaining follow up period. Epilepsy itself accounted for $21 \%$ of the mortality (11 and 73 deaths respectively) throughout the follow up period. Accidents accounted for 13\% (seven deaths) in the first 2 follow up years and $11 \%$ (37 deaths) thereafter.

To estimate the absolute risk of epilepsy related death we calculated the mortality rate directly. Some 18 of the 53 deaths in the first 2 years after diagnosis accounted for a mortality rate of 6.8 per 1000 person-years (95\% CI 4.1-10.7). After 2 years 110 of the 351 deaths could be attributed to epilepsy itself which accounted for a mortality rate of 3.1 per 1000 person-years (95\% CI 2.5-3.6).

Underlying causes of epilepsy accounted for $21 \%$ of the mortality (20 of 95 deaths) in the under 20 age group, In the older age group, this percentage dropped to $14 \%$ (42 of 309 deaths). Respiratory diseases were an important cause of death in the younger age group (15\% mortality, 14 deaths) but not in the older age group ( $4 \%$ mortality, 13 deaths). Epilepsy accounted for $26 \%$ of the mortality (25 deaths) in the under 20 age group, declining to $19 \%$

Table 4 Deaths in epilepsy by cause according to follow up and age at diagnosis

\begin{tabular}{|c|c|c|c|c|c|c|c|c|c|c|c|c|}
\hline & \multicolumn{3}{|c|}{ Follow up $<2 y$} & \multicolumn{3}{|c|}{ Follow up $>2 y$} & \multicolumn{3}{|c|}{ Age at diagnosis $<20 y$} & \multicolumn{3}{|c|}{ Age at diagnosis $>20 y$} \\
\hline & $O b s$ & $S M R$ & $95 \% C I$ & Obs & $S M R$ & $95 \% C I$ & $O b$. & $S M R$ & $95 \% C I$ & $O b s$ & $S M R$ & $95 \% C I$ \\
\hline All causes & 53 & 16 & $12-20$ & 351 & 2.8 & $2.5-3.1$ & 95 & 24 & $19-29$ & 309 & 2.5 & $2.2-2.8$ \\
\hline All neoplasms & 5 & 5.6 & $1.7-12$ & 70 & 1.7 & $1.3-2.1$ & 2 & 3.5 & $0.3-10.2$ & 73 & 1.8 & $1.4-2.2$ \\
\hline Malignant non-CNS & 1 & 1.3 & & 58 & 1.5 & $1.1-1.9$ & 1 & 0.7 & & 58 & 1.5 & $1.2-2.0$ \\
\hline Malignant CNS neoplasms & 3 & 75 & & 6 & 5.4 & $1.9-11$ & 0 & - & & 9 & 8.3 & $3.7-15$ \\
\hline Malignant respiratory & & & & & & & & & & & & \\
\hline neoplasms & 0 & - & & 18 & 1.9 & $1.1-2.9$ & 0 & - & & 18 & 1.9 & $1.1-2.9$ \\
\hline Respiratory diseases & 5 & 29 & $9.0-62$ & 23 & 3.8 & $2.4-5.6$ & 14 & 88 & $47-140$ & 13 & 2.1 & $1.1-3.5$ \\
\hline Pneumonia & 4 & 80 & $20-180$ & 13 & 8.8 & $4.6-14$ & 10 & 125 & $58-217$ & 7 & 4.9 & $1.9-9.2$ \\
\hline Cardiovascular diseases & 5 & 7.0 & $2.2-15$ & 64 & 1.5 & $1.1-1.9$ & 2 & 15 & $1.3-45$ & 67 & 1.5 & $1.2-1.9$ \\
\hline Ischaemic heart diseases & 1 & 2.6 & & 27 & 1.2 & $0.8-1.6$ & 0 & - & & 28 & 1.2 & $0.8-1.7$ \\
\hline Cerebrovascular diseases & 1 & 6.3 & & 22 & 2.5 & $1.6-3.7$ & 1 & 14 & & 22 & 2.5 & $1.5-3.7$ \\
\hline CNS diseases & 17 & 155 & $89-239$ & 97 & 42 & $34-51$ & 44 & 200 & $144-265$ & 70 & 32 & $25-40$ \\
\hline CNS diseases non-epilepsy & 6 & 67 & $23-132$ & 24 & 12 & $7.7-18$ & 19 & 112 & $66-169$ & 11 & 5.9 & $2.9-9.9$ \\
\hline Epilepsy & 11 & - & - & 73 & - & - & 25 & - & - & 59 & - & - \\
\hline All external causes & 8 & 10.5 & $4.4-20$ & 44 & 3.5 & $2.5-4.6$ & 11 & 5.4 & $2.6-9.1$ & 41 & 3.6 & $2.6-4.9$ \\
\hline Suicide & 1 & 13 & & 6 & 1.7 & $0.6-3.4$ & 1 & 11 & & 6 & 1.7 & $0.6-3.4$ \\
\hline All accident & 7 & 10.4 & $4.0-20$ & 37 & 4.3 & $3.0-5.9$ & 10 & 5.2 & $2.4-9.0$ & 34 & 4.6 & $3.2-6.4$ \\
\hline Traffic accident & 3 & 6.4 & $1.1-16$ & 15 & 2.5 & $1.4-4.0$ & 2 & 1.3 & $0.1-3.9$ & 16 & 3.3 & $1.9-5.1$ \\
\hline
\end{tabular}

International classification of diseases (9th revision): all causes=0001-9999; all neoplasms=1400-2399; malignant non-CNS=1400-1909/1920-2089; malignant CNS neoplasms $=1910-1919$; malignant respiratory neoplasms $=1620-1629$; respiratory diseases $=4600-5199$; pneumonia $=4800-4860$; cardiovascular diseases $=3900-4599$; ischaemic heart diseases $=4100-4149$; cerebrovascular diseases $=4300-4389$; CNS diseases $=3200-3499$; CNS diseases non-epilepsy $=3200-$ 3449/3460-3499; epilepsy=3450-3459; all external causes=E8000-E9990; suicide=E9500-E9590; all accident=E8000-E9499; traffic accident=E8000-E8199/ E8260-E8489.

Obs=Observed number of deaths; $S M R=$ observed number of deaths divided by expected number of deaths; numbers can exceed totals as subgroups can overlap. 
(59 deaths) in the older age group. Accidents accounted for $13 \%$ of mortality (10 deaths) in the under 20 age group and for $11 \%$ (34 deaths) in the older age group.

Seven deaths were attributable to suicide, six occurring both in the 2-41 year follow up period, and in the over 20 age group. In our study there was one violent death.

\section{Discussion}

Mortality in this cohort of 1355 incident epileptic patients (including all diagnostic classification types in an outpatient setting) was three times that of the Dutch population. As the mortality ratio in the local Heemstede region was only slightly less than for the total cohort, a possible selection of patients in our study could not have overtly distorted the outcome. It furthermore illustrates that the Instituut voor Epilepsiebestrijding in Heemstede at that period was a clinic with a low referral threshold.

Other studies comprising incident noninstitutionalised epileptic patients - namely the Rochester and NGPSE cohorts ${ }^{10}{ }^{13}$ - give risk ratios ranging from 2.1 to 3.0. In these studies the median age at onset was over 25 years and 36 years respectively, and included relatively few patients with an age at onset under 20 years, and more patients over 50 years of age. This contrasts with data from other demographic studies on epilepsy, ${ }^{21}{ }^{22}$ in which about $60 \%$ of all patients had a first seizure before the age of 20 . In the present study $60 \%$ of all patients are aged less than 20 years; this would seem a good reflection of the epilepsy population in general. However, given the changing demography of epilepsy-namely the decrease in incidence in young and the increasing incidence in elderly people-the time period we studied may no longer be representative, at least in developing countries.

Excess mortality was not only high in patients with onset of epilepsy before 20 years of age, but also in the first years after diagnosis. Compared with the general population, the mortality ratio was increased 16 -fold in the first 2 years of follow up, decreasing steadily thereafter. After a follow up of 15 years the mortality ratio showed a constant twofold increase, with a decrease to less than 1 after 30 years. Although most studies have a follow up period of less than 30 years, they show a similar trend to that found in the intermediate years in our study.

\section{CAUSES OF DEATH}

The underlying causes of epilepsy have been implicated as the cause for this increased mortality, rather than the epilepsy itself. Data from recent mortality studies on epilepsy suggest that underlying causes of disease are responsible for the increased mortality ratios in the first decade of life and the first few years after diagnosis. ${ }^{10} 13$ Nevertheless, data from our study indicate that epilepsy itself contributes to the increased mortality. Up to $20 \%$ of the mortality in patients with newly diagnosed epilepsy during the first 2 years after diagnosis is directly related to epilepsy. However, the abso- lute risk of dying from epilepsy in the first 2 years after the diagnosis has been made is relatively low. From the mortality rates it can be recalculated that roughly one out of 100 newly diagnosed patients with epilepsy will die from epilepsy itself.

Using causes of death as an end point of a study has its limitations as some death certificates could be misclassified, and sudden death in epilepsy cannot be established. Another approach would be the use of hospital records and those of general practitioners, in combination with witness accounts, to obtain more specific causes of death. By design, such an approach is impossible to use in this study. Deaths in our patients with epilepsy occurred from their 50 s to their 90 s. Using both death certificates and clinical information, which are available for a large proportion of our patients, is not likely to improve the validity of the study. This clinical information is undoubtedly unevenly distributed over patients and time, and would seriously distort the outcomes of the study.

The mortality risk from epilepsy in the first 2 years was as high as the mortality from underlying causes, and was consistent in the remainder of the follow up period. Despite the care that was taken to exclude patients with remote symptomatic unprovoked seizures, this high mortality may reflect a subgroup similar to the congenital neurodeficit group found in other epidemiological studies. $^{10172319}$ If we also included causes of death indirectly related to epilepsy, such as accidental deaths and suicides, the proportion of epilepsy related death increases, and is larger than the proportion of deaths related to the underlying causes, malignant CNS neoplasms, cerebrovascular diseases, and CNS diseases other than epilepsy. This has been reported in many of the older studies. $^{24} 25$

Mortality from malignant neoplasms excluding tumours of the CNS increased the excess occurring in the older age groups and in the follow up period of $2-41$ years. This indicates that undiagnosed cancer was unlikely to have been a cause of epilepsy in the patients included in the cohort, as mortality from this cause occurs long after diagnosis.

The mortality risk for all accidents was greatly increased, predominantly later in the follow up and in the older age groups. This might reflect the supervision of children and youngsters with epilepsy, as opposed to the more independent adult. The mortality risk for suicide showed a similar trend; however, the numbers involved are rather small. The mortality ratio for traffic (motorised and bicycle) accidents was also increased, again, predominantly in the older age groups, and in the later stages of follow up. In this older age group, the mortality in men was higher than in women. This is possibly distorted by the fact that more men hold a driver's license than women do.

The predisposing or underlying causes of epilepsy have always been reported as the predominant cause of death in the disease. Many studies have reported that around $20 \%$ of all deaths were attributable to the epilepsy. ${ }^{2}{ }^{7-12} 2425$ 
However, many of these studies included prevalent epilepic patients, mainly of older ages, and could therefore not give an adequate answer as to the mortality risk attributable to epilepsy. Our study of incident epileptic patients, however, gives results that are similar to these older studies, suggesting that epilepsy indeed carries a mortality risk in itself, albeit low. This excess mortality is more evident in the younger age groups and shortly after diagnosis.

This study was made possible by a grant from the Dutch Commissie Landelijke Epilepsie Onderzoek (CLEO); Subsidy: NEF/CLEO A-101. We thank the medical and administrative staff of the Instituut voor Epilepsiebestrijdin' for giving us access to the archives and outpatient clinics of the Institute and professor Dr H Meinardi for critical reading. We also thank the Department of Health Statistics of the Netherlands Central Bureau of Statistics in Voorburg, The Netherlands for use of their mortality statistics and database coupling. Special thanks are due to Mrs L Velmans for compiling the database and to Dr A de Boer for his initial ideas.

1 Gowers WR. Epilepsy and other chronic convulsive diseases: their causes, symptoms and treatment. New York: Dover Publications, republished 1964.

2 Munson JF. Death in epilepsy. Med Record 1910;77:58-62.

3 Massey EW, Schoenberg BS. Mortality from epilepsy: international patterns and changes over time. Neuroepidemiology 1985;4:65-70.

4 Chadwick D. Epilepsy. I Neurol Neurosurg Psychiatry 1994;57:264-77.

5 Malzberg B. Mortality among patients with mental disease. Ualzberg B. Mortality among patients wi

6 Alström CH. A Study of epilepsy in its clinical, social and Alström CH. A Study of epilepsy in its clinical, social and
genetic aspects. Uppsala: Appelbergs Boktryckeriaktiebolag genetic aspects.

7 Brink Henriksen P, Juul-Jensen P, Lund M. The mortality of epileptics. Epilepsy and insurance. London: Internationa Bureau for Epilepsy, 1967. (Social studies on epilepsy No 5.)

8 Zielinski JJ. Epilepsy and mortality rate and cause of death. Epilepsia 1974;15:191-201.
9 White SJ, McLean AEM, Howland C. Anticonvulsant drugs and cancer: A cohort study in patients with severe epilepsy. Lancet 1979;ii:458-61.

10 Hauser WA, Annegers JF, Elveback LR. Mortality in patients with epilepsy. Epilepsia1980;21:399-412.

11 Lühdorf K, Jensen KL, Plesner AM. Epilepsy in the elderly: life expectancy and causes of death. Acta Neurol Scand 1987;76:183-90.

12 Klenerman P, Sander JWAS, Shorvon SD. Mortality in patients with epilepsy: a study of patients in long term residential care. I Neurol Neurosurg Psychiatry 1993;56:149-52.

13 Cockerell OC, Johnson AL, Sander JWAS, et al. Mortality from epilepsy: results from a prospective population-based study. Lancet 1994;344:918-21.

14 Nashef L, Fish DR, Sander JWAS, et al. Incidence of sudden unexpected death in an adult outpatient cohort with epilepsy at a tertiary referral center. 7 Neurol Neurosurg Psyepilepsy at a tertiary refert
chiatry 1995;58:462-4.

15 Nilsson L, Tomson T, Farahmand BY, et al. Cause-specific mortality in epilepsy: a cohort study of more than 9000 patients once hospitalized for epilepsy. Epilepsia 1997;38: 1062-8.

16 Commission on Classification and Terminology of the International League Against Epilepsy. Proposal for revised classification of epilepsies and epileptic syndromes. Epilepsia 1989;30:389-99.

17 Sander JWAS, Shorvon SD. Epidemiology of the epilepsies. f Neurol Neurosurg Psychiatry 1996;61:433-43.

18 Penfield W, Jasper H. Epilepsy and the functional anatomy of the human brain. 1st ed. Boston: Little Brown, 1954.

19 Hauser WA, Hesdorffer DC. Mortality. Epilepsy: frequency, causes and consequences. New York: Demos, 1990:297-326.

20 Nashef L, Sander JWAS, Shorvon SD. Mortality in epilepsy. In: Pedley TA, Meldrum BS, eds. Recent advances in epilepsy. New York: Churchill Livingston, 1995:271-87.

21 Cockerell OC, Eckle I, Goodridge DMG, et al. Epilepsy in a population of 6000 re-examined: secular trends in first attendance rates, prevalence and prognosis. 7 Neurol Neurosurg Psychiatry 1995;58:570-6.

22 Pond DA, Bidwell BH, Stein L. A survey of epilepsy in 14 general practices. I. Demographic and medical data. Psychiatr Neurol Neurochir 1960;63:217-36.

23 Sander JWAS. Some aspects of prognosis in the epilepsies: a review. Epilepsia 1993;34:1007-16.

24 Krohn W. Causes of death among epileptics. Epilepsia 1963;4:315-21

25 Iivanainen $M$, Lehtinen J. Causes of death in institutionalized epileptics. Epilepsia 1979:20:485-92. 